\title{
Discussion en face à face ou en ligne
}

\section{Daniel Schröpfer}

Dr méd., médecin de la ville de Zurich, membre du Comité de l'Association des médecins dirigeants d'hôpitaux de suisse (AMDHS), membre de la rédaction

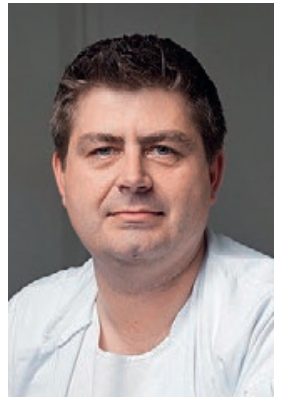

Ces dernières semaines ont nourri l'espoir de pouvoir à nouveau se retrouver plus souvent dans la sphère privée et professionnelle. Or pour l'heure, l'augmentation importante du nombre de cas quotidien montre qu'il nous reste encore un bon bout de chemin à parcourir. Mesures et plans de protection restent de mise pour maîtriser la situation et tendre vers la «normalité».

La situation qui prévalait l'an dernier a entraîné de nombreux changements, notamment en matière d'utilisation de formats électroniques de communication. Parallèlement à mon activité en contact direct avec les patients, j'ai pu participer à de nombreuses réunions en ligne. Il n'y a pas que les connaissances techniques nécessaires, mais aussi l'affinité personnelle pour ce type d'exercice qui représentent, d'après moi, des obstacles - surmontables. J'ai découvert avec étonnement que même en appliquant rigoureusement les consignes pour rejoindre une réunion en ligne, l'accès m'était refusé, quand bien même je suis l'organisateur de la séance. J'ai aussi constaté l'incompatibilité des systèmes de différentes institutions. Se connecter à temps, bien enclencher la caméra et le microphone, sans oublier le choix de l'arrière-plan: nous avons tous et toutes expérimenté les défis liés au démarrage d'une réunion.

Les réunions de plus de quatre heures sans interruption, baisse de concentration et accès de bâillements inclus, ont constitué pour moi un réel challenge. Franchement, qui, en temps normal, aurait l'idée de

\section{Les séances en ligne ont l'avantage de nous} épargner les trajets, même si les échanges y sont moins spontanés que dans la vraie vie.

prévoir une séance si longue et sans pauses? Nous en avons aussi tiré les leçons: les réunions marathon font heureusement désormais partie du passé.

Il est vrai que les séances en ligne ont l'avantage de nous épargner les trajets, même si les échanges y sont moins spontanés et interactifs que dans la vraie vie. Pour certaines professions et dans certaines situations, il est évident que les sessions de formation postgraduée et continue en ligne amènent une réelle plus-value, même si la possibilité de corriger en direct ne doit pas être sous-estimée lors d'échanges personnels.
Lors d'une récente discussion avec la direction d'une faculté, j'ai réalisé que dans les universités et les hautes écoles, certaines étudiantes et certains étudiants n'auront connu qu'un enseignement virtuel et suivi trois à quatre semestres uniquement en ligne. Qu'en est-il des échanges avec les autres, de l'apprentissage en groupe, des interactions sociales? Cela ne présage rien de bon pour la jeune génération.

L'année dernière, un grand nombre de sessions de formations postgraduées et congrès médicaux se sont déroulés en ligne. Cela ne me pose pas de problèmes si ce n'est que quelques fois, mais dans l'ensemble le contact direct me manque. J'ai pu participer à deux manifestations, en septembre 2020 et en juillet 2021: j'aimerais ici remercier les organisatrices et les organisateurs de ces événements avec concepts de protection. Un réel bonheur de se voir en vrai et surtout que ce soit faisable.

\section{La communication électronique ne concerne} cependant pas que les réunions en ligne, mais aussi les échanges de courriels.

La communication électronique ne concerne cependant pas que les réunions en ligne, mais aussi les échanges de courriels. Avez-vous déjà compté le nombre de newsletters ou de suggestions de lecture que vous recevez quotidiennement dans votre boîte mail? Combien en lisez-vous ou en survolez-vous? Un grand nombre de revues (médicales) sont désormais aussi disponibles en ligne. Un avantage considérable pour une équipe répartie sur différents sites: plus besoin de porter des piles de revues d'un endroit à l'autre, sans compter la plus-value pour l'environnement d'une version électronique. Et si un article vous intéresse particulièrement, vous avez toujours la possibilité de l'imprimer. De plus, avec les courriels, chacun peut décider s'il souhaite l'effacer sans le lire. Et pourtant, je ressens néanmoins le besoin de tenir un journal, une revue ou un livre entre les mains pour le simple plaisir de le feuilleter. J'ai déjà lu des livres électroniques, mais cela reste étrange et inhabituel.

Et vous, chères lectrices et chers lecteurs, qu'en pensez-vous? Lisez-vous l'édition numérique du Bulletin des médecins suisses de la même manière que la version papier? Etes-vous attachés au papier ou seriez-vous enclins à appuyer plus souvent sur la touche delete? 